Polymer Journal, Vol. 5, No. 1, pp 72-85

\title{
Morphological Studies of Cold Drawn Poly(ethylene terephthalate)
}

\author{
Tsutomu AsAno \\ Department of Physics, Faculty of Science, \\ Shizuoka University, Shizuoka, Japan \\ Tsuneo Seto \\ Department of Physics, Faculty of Science, \\ Tokyo Metropolitan University, Setagaya-ku, \\ Tokyo, Japan.
}

(Received January 16 1973)

\begin{abstract}
Morphology of cold drawn poly(ethylene terephthalate) (PET) was studied by the X-ray diffraction method. In slowly drawn PET, paracrystalline reflections were observed at an early stage of crystallization by annealing, and it was found that the paracrystalline structure was basically monoclinic. In the case of annealing at temperatures below $100^{\circ} \mathrm{C}$, the crystalline structure became triclinic after passing through the paracrystalline state. Tilting of the crystalline $c$-axis (molecular axis) was seen in the course of the transition from the paracrystalline to the triclinic state. At higher annealing temperatures, the triclinic crystallites were sufficiently developed to form crystalline layers in which the molecular chains were tilted in directions which depended on the inclination of the layers.

In the case of rapid drawing, strain-induced crystallization occurred, but paracrystalline reflections were not observed. In the rapidly drawn sample, tilt of the $c$-axis was small, while inclination of the layer was large.

KEY WORDS Poly(ethylene terephthalate) / X-ray / Cold Drawing / Paracrystal / Tilted Orientation / Layer Structure /
\end{abstract}

It is generally accepted that quenched poly(ethylene terephthalate) (PET) is a glassy amorphous substance, and that cold drawing of quenched PET samples produces an oriented material containing little crystalline matter. The crystallinity of the samples gradually increases upon annealing. Therefore, the cold drawn sample is suitable for a study of crystallization from the amorphous state. The morphology of drawn PET has been studied by several authors. In the case of drawing above the glass transition temperature, Bonart ${ }^{1}$ found that it was possible to produce an intermediate paracrystalline structure. He reported that the structure of the sample varies from totally amorphous to a nematic and then a smectic state during the drawing treatment. From electron microscopic observation, Yeh and $\mathrm{Geil}^{2,3}$ pointed out that a glassy PET material is composed of ball-like structures in which mole- cules are in paracrystalline order, and that straininduced crystallization is caused by rotation, alignment, and perfection of the internal order of the paracrystalline ball-like structures.

In the wide angle X-ray diffraction pattern of drawn PET, reflections are often displaced, some up and some down, from normal layer line positions, which shows not only that the molecular chain axes of crystals are tilted with respect to the draw axis, but further that they are all tilted by about the same amount and in the same direction with respect to the unit cell. Thus, some definite crystallographic axis, which deviates slightly from the crystalline $c$-axis, is parallel to the draw axis. This type of orientation is known as the tilted orientation, and was first studied in PET by Daubeny, Bunn, and Brown. ${ }^{4}$ They reported that crystals in PET fibers, drawn at $75^{\circ} \mathrm{C}$ and annealed at $210^{\circ} \mathrm{C}$, 
are tilted by about $5^{\circ}$ in such a precisely defined direction that the crystallographic ( $\overline{2} 30)$ plane remains vertical and the inclination of the (001) plane to the draw axis increases. Bonart ${ }^{5}$ explained that the tilt of PET results from an inclined layer structure with the molecular axes having opposite tilts in the alternating crystalline and amorphous layers. He indicated that the above mentioned $(\overline{2} 30)$ orientation is realized when the surface of the crystalline layer coincides with a crystallographic plane such as (112) or (111).

In our preliminary experiments, some new information about the paracrystalline structure was obtained in the samples annealed at low temperatures. In particular, the tilt direction varied with annealing temperature, which disagrees with the observations by Daubeny, et al., ${ }^{7}$ and by Bonart. ${ }^{5}$ Therefore, it seemed interesting to investigate the super structural change during the crystallizing process. In this work, cold drawn PET samples were studied by wide and small angle $\mathrm{X}$-ray diffraction as functions of drawing speed, annealing temperature, and annealing time. Detailed investigations were made on the paracrystalline structure and tilt of the crystalline $c$-axis.

\section{EXPERIMENTS}

Samples were prepared from poly(ethylene terephthalate) manufactured by Teijin Co., Ltd.. Preliminary X-ray work showed that the PET fiber, quenched in ice water from the melt, gave a diffuse uniform ring which is characteristic of noncrystalline substances.

The glassy PET samples were drawn through the necking stage at $20^{\circ} \mathrm{C}$. Appearance of the drawn samples changed with the drawing speed. The sample drawn at the rate of $10.0 \mathrm{~mm} / \mathrm{sec}$ was white and opaque, whereas the one drawn at $0.1 \mathrm{~mm} / \mathrm{sec}$ was transparent. Effect of the drawing speed on morphology was examined by comparing the samples drawn under the two conditions.

The drawn samples, kept in a taut state to maintain their length constant, were annealed in a silicon oil bath. Then they were observed by wide and small angle $\mathrm{X}$-ray diffraction at room temperature.

\section{RESULTS}

$X$-ray Diffraction Data of Slowly Drawn Samples

Cold Drawing. The wide-angle X-ray pattern, obtained from the sample drawn at $20^{\circ} \mathrm{C}$ at the rate of $0.1 \mathrm{~mm} / \mathrm{sec}$, shows very broad scattering peaks on the equator, which indicate a uniaxial orientation of the amorphous molecules. As no crystalline reflections exist in this pattern, straininduced crystallization probably did not occur during the drawing treatment. Slowly drawn samples were annealed at various temperatures from $40^{\circ} \mathrm{C}$ to $250^{\circ} \mathrm{C}$.

Annealing at $40^{\circ} \mathrm{C}$. Figure $1 \mathrm{a}$ is a pattern given by a sample annelaed at $40^{\circ} \mathrm{C}$ for $10^{4} \mathrm{sec}$. Weak crystalline reflections, whose spacings are about $10.3 \AA$, appear on the meridian. Since the spacing is approximately equal to the length of the monomer unit, these reffections are probably given by the (001) plane. The reflections seem to correspond to those reported by Bonart ${ }^{1,6,7}$ as reflections from a paracrystalline state. He observed meridional reflections, whose spacing was about $10.7 \AA$, in the sample drawn above the glass transition temperature. The meridional reflections indicate the existence of a paracrystalline order which is different from the usual triclinic form.

The equatorial broad peaks were changed slightly by the annealing. The insides of the peaks, which correspond to smaller diffraction angles, split into two parts situated above and below the equator (see arrow in Figure 1a). This fact suggests that molecules are tilted by the annealing.

Annealing at $60^{\circ} \mathrm{C}$. In samples annealed at $60^{\circ} \mathrm{C}$, which is just below the glass transition temperature, sharp crystalline reflections were observed on the meridian. The sample annealed at $60^{\circ} \mathrm{C}$ for $10^{4} \mathrm{sec}$ (Figure 1b) showed the strongest meridional peaks among those obtained in this work, provided that the samples were annealed for $10^{4} \mathrm{sec}$ or less at each temperature. The spacing of the reflections is about $10.3 \AA$.

In the sample annealed for $10^{4} \mathrm{sec}$, it was possible to divide the broad equatorial reflection into four peaks. The inner part of the reflection split into two diffuse peaks situated above and below the equator, which had already been observed in samples annealed at $40^{\circ} \mathrm{C}$. At the 


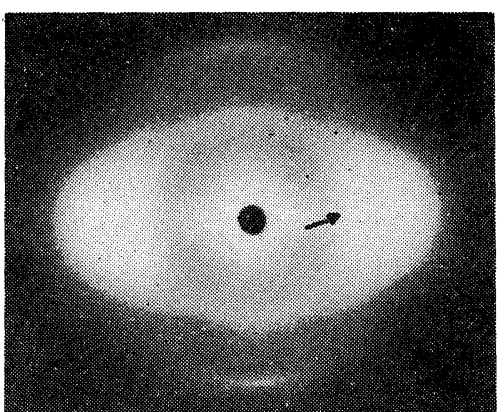

(a)

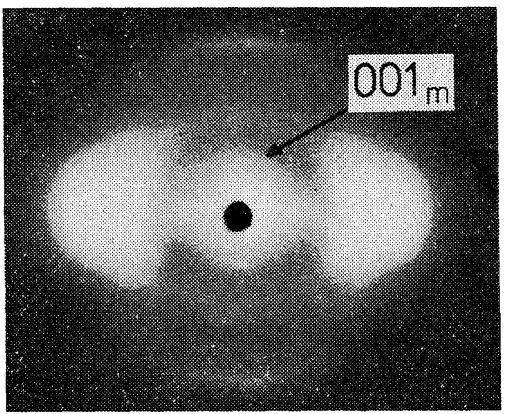

(b)
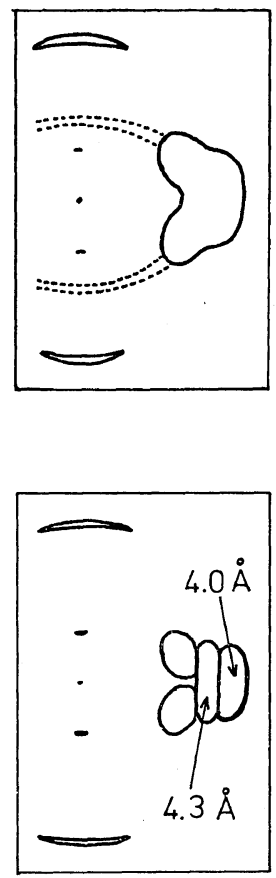

Figure 1. Wide angle X-ray diffraction patterns of PET fibers drawn at $0.1 \mathrm{~mm} / \mathrm{sec}$ : (a), annealed at $40^{\circ} \mathrm{C}$ for $10^{4} \mathrm{sec}$; (b), annealed at $60^{\circ} \mathrm{C}$ for $10^{4} \mathrm{sec}$.

center of the reflection, a strong peak appeared which shows a spacing of about $4.3 \AA$. The outer part gave a peak which shows a spacing of about $4.0 \AA$. By annealing at temperatures higher than $60^{\circ} \mathrm{C}$, the outer and inner peaks were gradually moved to the normal triclinic 100 and 010 reflections respectively, and their intensity grew simultaneously. Diffuse reflections from the triclinic structure were also observed on the first and second layer lines. These results suggest that well-developed paracrystalline parts and triclinic crystals coexist in the sample.

Effect of annealing time was also studied at $60^{\circ} \mathrm{C}$. In the case of annealing for $10 \mathrm{sec}$, the meridional reflection was diffuse, and the X-ray pattern resembled that given by the sample annealed at $40^{\circ} \mathrm{C}$ for $10^{4} \mathrm{sec}$ (Figure 1a). Intensity of the meridional 001 reflection increased with increase in annealing time. The intensity of the peaks appearing at the center of the diffuse reflections on the equator increased similarly, which suggests that the peaks were given by the paracrystalline form. The inner peaks appearing in the diffuse reflections on the equator moved slightly to the center of the pattern with increase in annealing time. This fact indicates that the spacing of the plane corresponding to the triclinic (010) expands in the early stage of annealing.

Annealing at $80^{\circ} \mathrm{C}$. The X-ray pattern of the sample annealed at $80^{\circ} \mathrm{C}$ for $10^{4} \mathrm{sec}$ shows a number of crystalline reflections (Figure 2a). Most of these reflections seem to be given by triclinic crystals. However, nontriclinic reflections still exist on both the meridian and the equator. The two weak reflections, whose spacings are about $4.3 \AA$ and $4.6 \AA$ respectively, are observed on the equator. Both reflections disappeared when the triclinic reflections were fully developed at higher annealing temperatures. The reflection of spacing $4.3 \AA$ is the same one as the strong peak observed in the sample annealed at $60^{\circ} \mathrm{C}$. The reflection of spacing $4.6 \AA$, which splits into two peaks above and below the equator, was not observed at $60^{\circ} \mathrm{C}$, since 


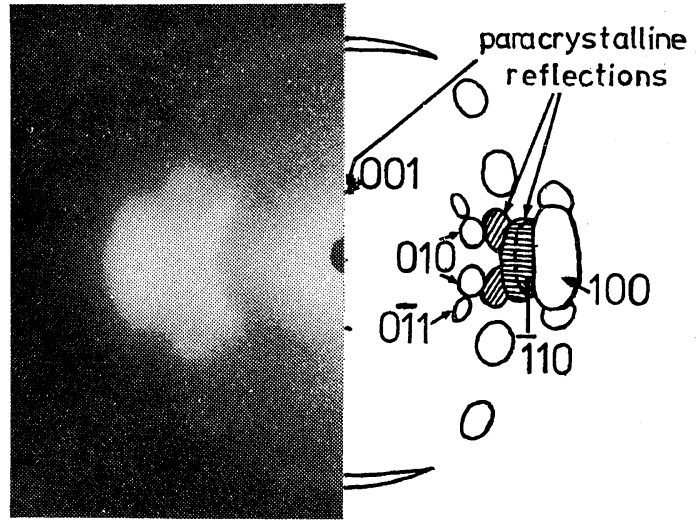

(a)

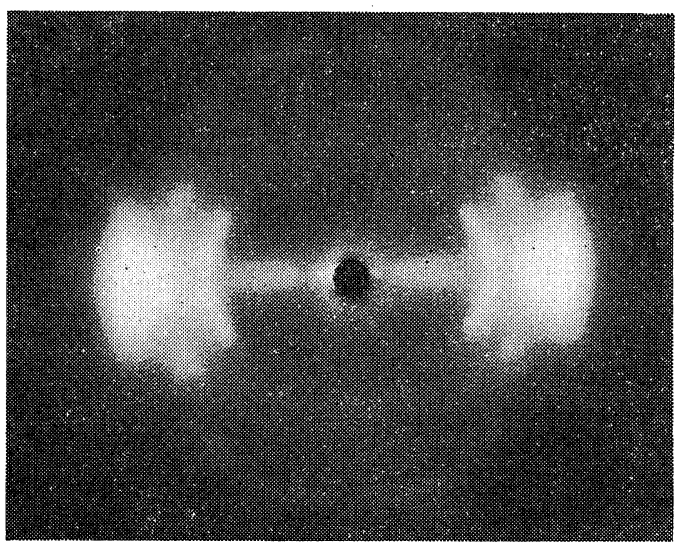

(b)

Figure 2. Wide angle $\mathrm{X}$-ray diffraction patterns of PET fibers: (a) annealed at $80^{\circ} \mathrm{C}$ for $10^{4} \mathrm{sec}$, (b) annealed at $100^{\circ} \mathrm{C}$ for $10^{4} \mathrm{sec}$. the triclinic 010 reflection overlapped on it.

From observation of samples annealed for various times, it was revealed that the intensity of the meridional 001 peak increases at the beginning and then decreases with development of the triclinic reflections. These results suggest that the crystallites become triclinic after passing through the paracrystalline state: i.e., the triclinic structure is not formed directly from the amorphous phase.

In Figure 2a, the triclinic $0 \overline{1} 1$ and 010 reflections are very sharp, while the other reflections are broad. The $0 \overline{1} 1$ is the sharpest. The sharp 010 splits into two separate maxima. The 100 peak is very broad. Considering that the breadth of these reflections is defined by the dimensions of the crystallites, the external form of the crystallite was estimated. The result shows that the triclinic crystallite grows in a direction nearly perpendicular to the $(0 \overline{1} 1)$ plane and hence that it has an extremely small dimension in the direction perpendicular to the (100) plane.

Annealing at $100^{\circ} \mathrm{C}$. The paracrystalline reflections disappeared gradually in proportion to the development of the triclinic reflections. The meridional 001 reflection and the equatorial paracrystalline reflections were no longer observed after annealing above $100^{\circ} \mathrm{C}$. In the pattern shown in Figure $2 \mathrm{~b}$, all the reflections are given by the triclinic crystal.

In order to examine the transition from the paracrystalline to the triclinic structure, the spacings of the triclinic reflections were measured

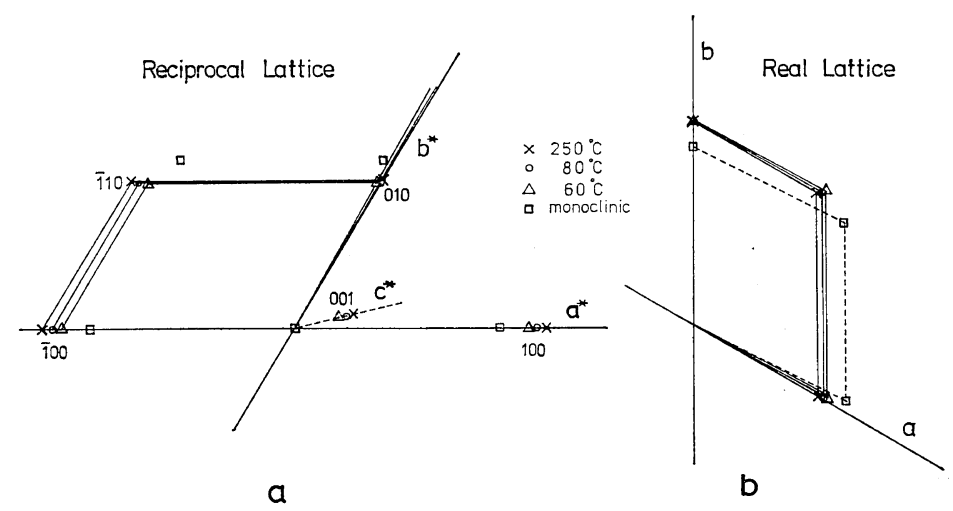

Figure 3. (a) Variation in the reciprocal lattice in $h k 0$ plane. $\times, \bigcirc$, and $\triangle$ represent the data from the samples annealed at $250^{\circ} \mathrm{C}, 80^{\circ} \mathrm{C}$, and $60^{\circ} \mathrm{C}$, respectively. (b) Corresponding variations in the real lattice (the $c$-axis projection.) 
from the patterns of the samples annealed at lower temperatures. Although the accuracy of the measurement was lowered with increase in the broadness of the spots, determination of both spacings and indices was possible in the patterns of the samples annealed above $60^{\circ} \mathrm{C}$.

On the basis of the observed spacings, the reciprocal lattice was formed for each annealing temperature (Figure 3a). The result shows that the triclinic reciprocal lattice is slightly changed by annealing at low temperatures. The reciprocal lattice expands in the direction of the $a^{*}$-axis with rise of annealing temperature, while the position of the 010 lattice point scarcely moves above $60^{\circ} \mathrm{C}$. (As observed at $60^{\circ} \mathrm{C}$, the spacing of the 010 reflection increases in the early stage of the transition from the paracrystalline state.)
The angle which the $c^{*}$-axis made with the $a^{*} b^{*}$ plane slightly decreases with rise of annealing temperature. This fact shows that the inclination of the triclinic (001) plane is increased gradually by annealing. The triclinic structure approaches stability and the variation in the reciprocal lattice stops at annealing temperatures above $150^{\circ} \mathrm{C}$. The corresponding variation in the real lattice is shown in Figure $3 b$, which shows that the lattice contracts mainly in the direction perpendicular to the (100) plane.

Annealing at Higher Temperatures. The wide angle patterns of the fibers annealed at $150^{\circ} \mathrm{C}$, $200^{\circ} \mathrm{C}$, and $250^{\circ} \mathrm{C}$, respectively, are shown in Figure 4. All the spacings given by these patterns agreed with those of the triclinic structure of Daubeny, et $a{ }^{4}{ }^{4}$ In these patterns, the
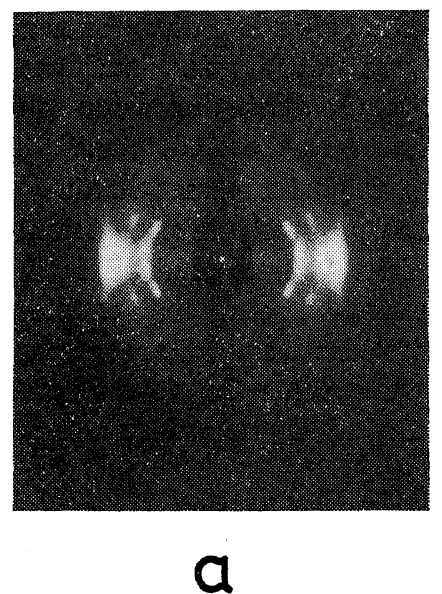

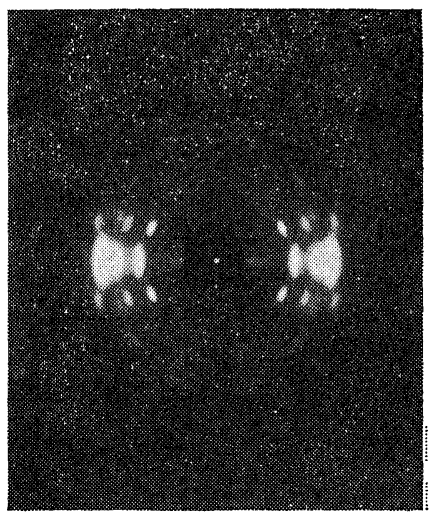

b

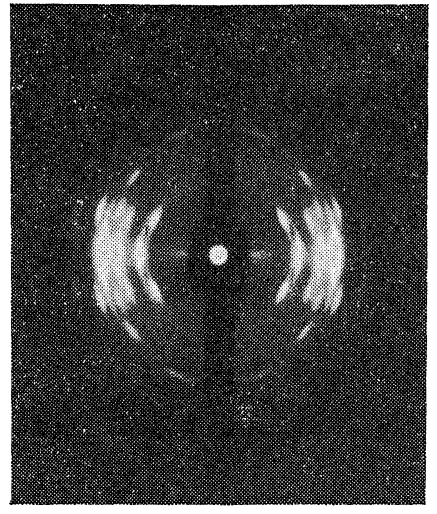

C

Figure 4. Wide angle $\mathrm{X}$-ray diffraction patterns of PET fibers. The annealing temperatures are (a) $150^{\circ} \mathrm{C}$, (b) $200^{\circ} \mathrm{C}$, and (c) $250^{\circ} \mathrm{C}$.
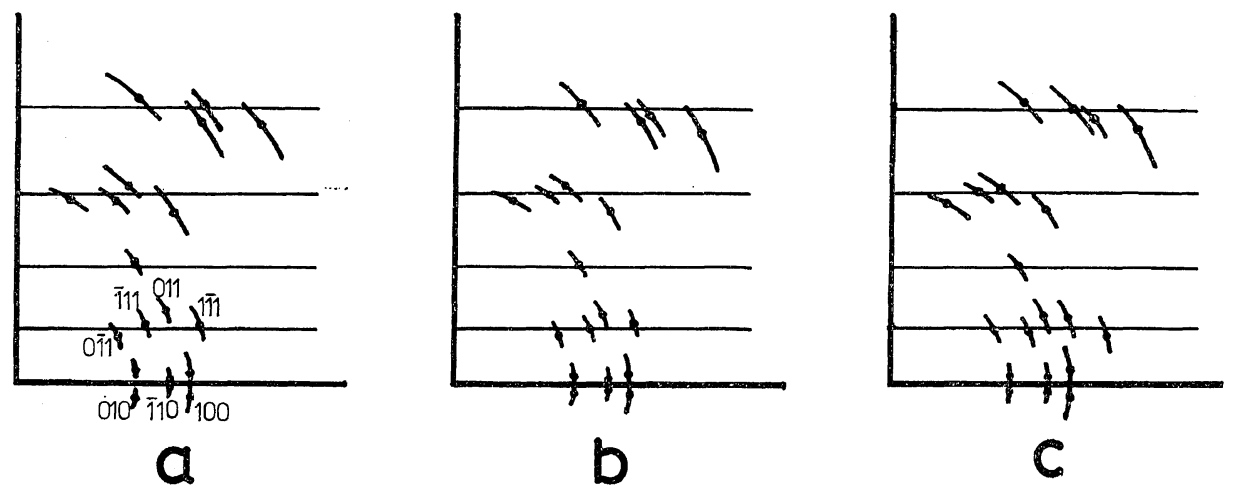

Figure 5. Displacement of the reflections. The arcs show the observed reflections shown in Figure 4 and the dots show the calculated positions. 
reflections are displaced from the normal layer lines, which shows that the molecular chain axes (c-axes) of the triclinic crystals are tilted. The displacement of each reflection varies with annealing temperature (Figure 5). Accordingly, the crystalline tilt varies with annealing temperature. This disagrees with Daubeny, et al., ${ }^{4}$ and with Bonart. ${ }^{5}$

It is possible to analyse the X-ray patterns of drawn PET by a method which has been developed for the tilted orientation. ${ }^{8}$ Figure 6 represents the reciprocal lattice net in the $a^{*} b^{*}$ plane together with some lattice points of the first layer line projected on it. If we image the tilting of the $c$-axis about a line lying in the plane of paper and passing through the origin of the reciprocal lattice, some of the points would move downwards and others upwards from the plane of the paper. In Figure $4 c$, for example, the $0 \overline{1} 1$ and $\overline{1} 11$ reflections are displaced downwards, while the 011 and $1 \overline{1} 1$ ones move upwards from their normal positions. Therefore the lattice must be tilted by rotation about the line $\mathrm{AA}^{\prime}$ shown in Figure 6 . The height of any point from the plane of the paper is given by

$$
\zeta=z \cos \phi-x \sin \phi
$$

where $z$ is the height of the normal untilted reciprocal lattice layer, $\phi$ is the angle of tilt, and $x$ is the distance from the tilt line on the paper. If the tilt line and $\phi$ are calculated from the position of some reflections, values of $\zeta$ may be calculated for all the others, and these should agree with their heights form the equator in the photograph. As shown in Figure 5, all the tilt displacements, which are calculated from displacements of the $0 \overline{1} 1,011, \overline{1} 11$, and $1 \overline{1} 1$ spots, agree with those of the diffraction pattern.

In Figure 7, the tilt of the crystalline $c$-axis is illustrated with the aid of the real unit cell.

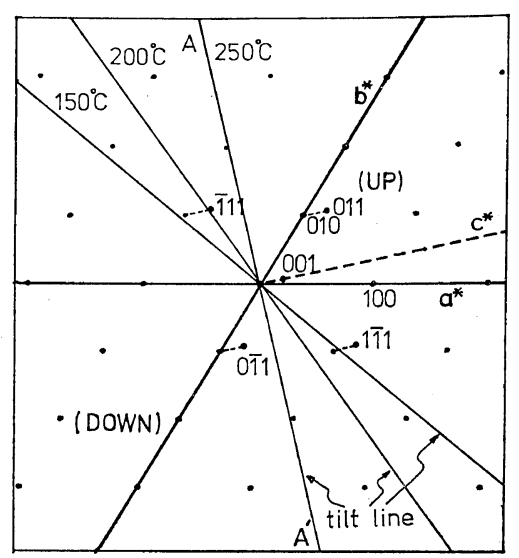

Figure 6. The reciprocal lattice net and the tilt lines.
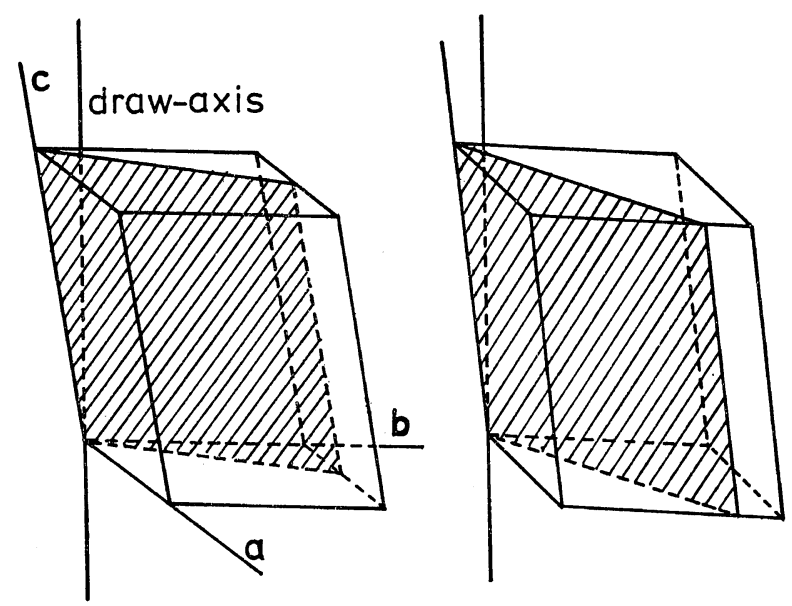

a

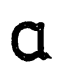

Figure 7. Tilt of the crystalline $c$-axis. The azimuth of the tilt varies with annealing temperature.
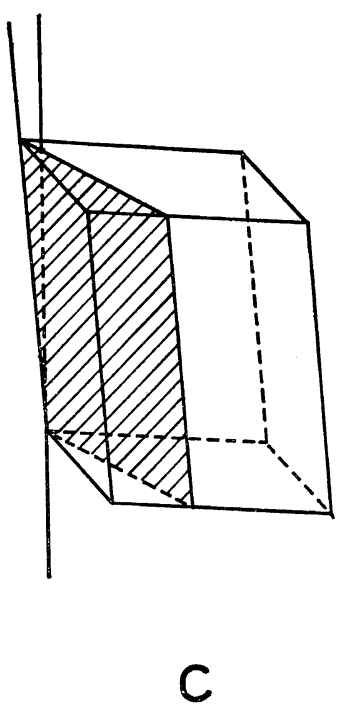


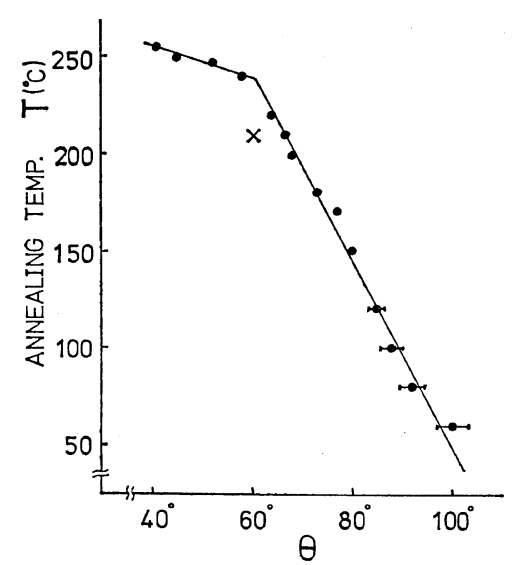

Figure 8. Annealing temperature vs. azimuth of the crystalline tilt, where $\theta$ is defined by the angle between the tilt line and the $b^{*}$-axis.

The relation between the direction of the tilt line and the annealing temperature is shown in Figure 8 , where $\theta$ represents the angle between the tilt line and the $b^{*}$-axis in the reciprocal basal plane. The cross in Figure 8 indicates the value reported by Daubeny, et al., and by Bonart. (This value was measured with the sample drawn at a temperature above the glass transition.) As the triclinic lattice varied at low temperatures, tilting of the crystal was analysed by using the changed reciprocal lattice. The value of $\theta$ varies almost continuously from $100^{\circ}$ to $40^{\circ}$ with rise of annealing tempetarure. The slope of the $\theta-T$ curve changes at about $T=240^{\circ} \mathrm{C}$. Tilting always occurs in such a way that inclination of (001) plane to the draw axis decreases, which is contrary to the data reported by Daubeny, et al., and by Bonart. Tilt angle $\phi$ is about $8^{\circ}$ in low temperatures and about $5^{\circ}$ in high temperatures. The experimental results are summarized in Table I.

\section{$X$-ray Observation of Rapidly Drawn Samples}

In the X-ray pattern of a fiber drawn at 10.0 $\mathrm{mm} / \mathrm{sec}$, crystalline reflections appeared before annealing. As shown in Figure 9a, two fairly strong reflections whose indices are triclinic $0 \overline{1} 1$ and 010 are observed, together with several diffuse triclinic reflections. The difference in broadness of the triclinic reflections is not so remarkable as that observed in the slowly drawn samples. The 010 reflection concentrates on the

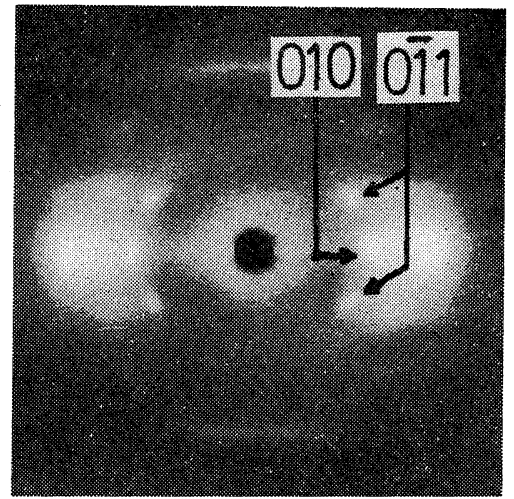

(a)

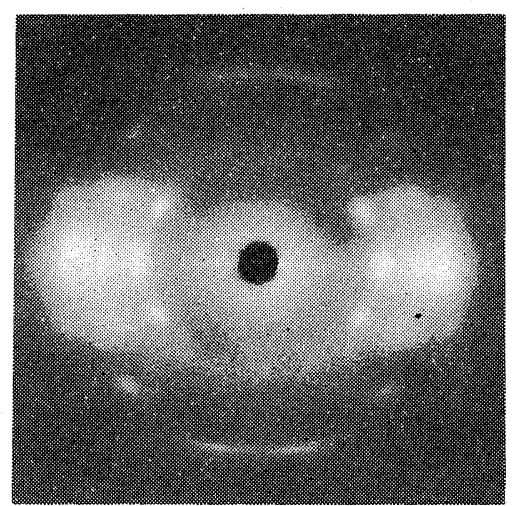

(b)

Figure 9. Wide-angle $\mathrm{X}$-ray diffraction patterns of fibers drawn at $10.0 \mathrm{~mm} / \mathrm{sec:}$ (a), before annealing; (b), annealed at $100^{\circ} \mathrm{C}$ for $10^{4} \mathrm{sec}$.

equator, hence the crystalline $c$-axis is nearly parallel to the draw axis. The features of the pattern were not greatly changed by annealing at temperatures below $100^{\circ} \mathrm{C}$ (Figure 9b). The paracrystalline reflections do not appear in the rapidly drawn samples.

In samples annealed at higher temperatures, tilting of the crystalline $c$-axis was observed. Azimuth of the tilt depended on annealing temperature in nearly the same way as that observed in the slowly drawn samples. The tilt angle is smaller than that of the slowly drawn samples. The results are given in Table I.

\section{Layer Structure}

The layer structure of drawn PET samples was studied by small angle $\mathrm{X}$-ray diffraction. Inclination of a layer, which is defined by the 
Morphology of Cold Drawn Poly(ethylene terephthalate)

Table I.

\begin{tabular}{|c|c|c|c|c|c|c|c|}
\hline \multicolumn{4}{|c|}{ Sample Treatment } & \multicolumn{2}{|c|}{ Wide-Angle X-Ray Data } & \multicolumn{2}{|c|}{ Small-Angle X-Ray Data } \\
\hline $\begin{array}{l}\text { Draw- } \\
\text { ratio }\end{array}$ & $\begin{array}{l}\text { Drawing } \\
\text { speed, } \\
\mathrm{mm} / \mathrm{sec}\end{array}$ & $\begin{array}{l}\text { Annealing } \\
\text { tempera- } \\
\text { ture, }{ }^{\circ} \mathrm{C}\end{array}$ & $\begin{array}{l}\text { Annealing } \\
\text { time, sec }\end{array}$ & $\begin{array}{l}\text { Azimuth of } \\
\text { the } \\
\text { crystalline } \\
\text { tilt } \theta \\
\text { degree }\end{array}$ & $\begin{array}{l}\text { Tilt angle } \\
\phi, \text { degree }\end{array}$ & $\begin{array}{l}\text { Inclination } \\
\text { of layer } \\
\text { degree }\end{array}$ & $\begin{array}{l}\text { Long } \\
\text { spacing, }\end{array}$ \\
\hline 4.0 & 0.1 & 60 & $10^{4}$ & 100 & $8 \sim 10$ & - & - \\
\hline 4.0 & 0.1 & 80 & $10^{4}$ & 92 & 8 & - & - \\
\hline 4.0 & 0.1 & 100 & $10^{4}$ & 88 & 7 & - & - \\
\hline 4.0 & 0.1 & 150 & $10^{4}$ & 80 & 5.0 & 55 & 116 \\
\hline 4.0 & 0.1 & 170 & $10^{4}$ & 77 & 3.9 & 46 & 120 \\
\hline 4.0 & 0.1 & 200 & $10^{4}$ & 68 & 4.7 & 42 & 130 \\
\hline 4.0 & 0.1 & 240 & $10^{4}$ & 58 & 4.7 & 35 & 148 \\
\hline 4.0 & 0.1 & 250 & $10^{3}$ & 45 & 5.0 & 25 & 165 \\
\hline 5.5 & 10.0 & 150 & $10^{4}$ & 80 & 1.5 & - & 113 \\
\hline 5.5 & 10.0 & 170 & $10^{4}$ & 70 & 1.0 & 60 & 130 \\
\hline 5.5 & 10.0 & 200 & $10^{4}$ & 68 & 1.5 & 59 & 134 \\
\hline 5.5 & 10.0 & 240 & $10^{4}$ & 60 & 1.0 & 45 & 154 \\
\hline 5.5 & 10.0 & 250 & $10^{3}$ & 52 & 1.0 & 40 & 154 \\
\hline
\end{tabular}

angle between the draw axis and the normal of the layer surface, decreases and long spacing increases with rise of annealing temperature.

In the samples drawn at $10.0 \mathrm{~mm} / \mathrm{sec}$, inclination of layers was larger than that of the slowly drawn samples. It was somewhat difficult to determine the inclination from the small angle pattern, since the peaks are spread along the layer line. The small-angle diffraction data are summarized in Table I.

\section{DISCUSSION}

\section{Paracrytsalline Structure}

In the wide-angle diffraction patterns of the slowly drawn PET samples, nontriclinic crystalline reflections were observed. The sharp meridional reflection, whose spacing is about $10.3 \AA$, indicated that the (001) plane of this crystalline form is perpendicular to the draw axis. The broad reflections appearing on the equator gave spacings of about $4.3 \AA$ and $4.6 \AA$. These paracrystalline reflections gradually disappeared with the development of triclinic reflections. The $\mathrm{X}$-ray diffraction studies under various annealing conditions revealed that, in the course of crystallization from the glassy state, most of the triclinic crystallites are formed through the par- acrystalline state.

The paracrystalline structure is determined from the data obtained in this experiment. In general, a crystalline polymer molecule forms three principal crystallographic planes, such as $\{100\},\{010\},\{110\}$, hence the three corresponding peaks should appear on the equator. In the case of drawn PET, the three lattice planes of the triclinic form are actually observed. Since the paracrystalline structure transforms to the triclinic one, the three principal planes of the triclinic form will correspond to those in the paracrystalline structure. As the paracrystalline structure gives only two reflections on the equator, two of the three principal planes seem to have equal spacing. From the movement of the reflections in the early stage of crystallization, the triclinic 010 spot seems to correspond to the paracrystalline reflection giving a lattice spacing of $4.6 \AA$. On the other hand, the triclinic 100 and $\overline{1} 10$ spots are near to the paracrystalline reflection showing a spacing of $4.3 \AA$. At low annealing temperatures, the two triclinic spots seem to merge into the broad reflection whose center showed the spacing of $4.3 \AA$. The triclinic and the paracrystalline reflections gradually separated from each other by annealing at higher temperatures. Therefore, it may be considered 


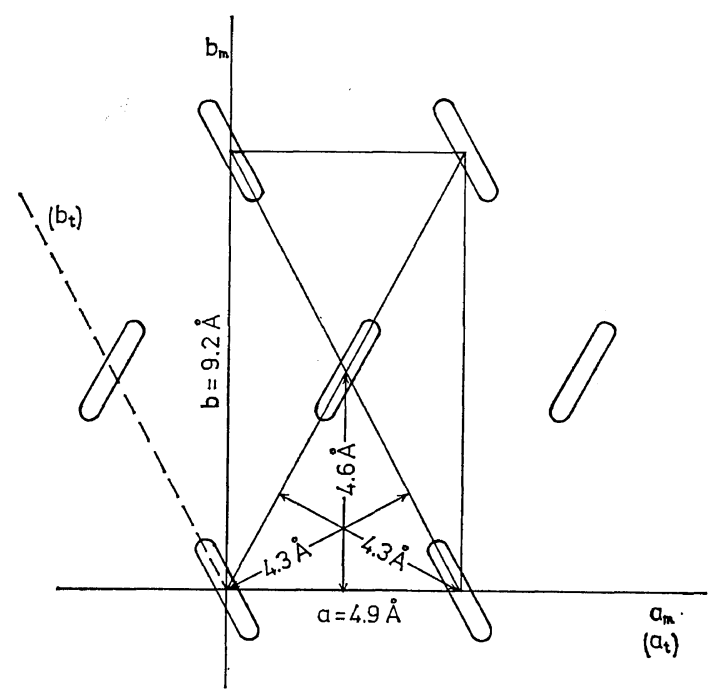

Figure 10. The paracrystalline unit cell and molecules (the $c$-axis projection). The $a_{\mathrm{m}}$ - and $b_{\mathrm{m}}$-axes represent the monoclinic lattice and the $a_{\mathrm{t}^{-}}$and $b_{t}$-axes represent the triclinic one transformed from the monoclinic form. The ellipse shows the direction of the benzene ring.

that the paracrystalline structures corresponding to the triclinic $(100)$ and $(110)$ planes have equal spacing of $4.3 \AA$. The two principal planes are nearly normal to the paracrystalline (001) plane, while the principal plane corresponding to the triclinic (010) plane is not normal to the (001) plane, since the peak of $4.6 \AA$ split into two parts. These facts are not explained by the hexagonal form proposed by Bonart. ${ }^{1}$ From the data, the paracrystalline structure is determined as base-centered monoclinic with lattice constants $a=4.9 \AA, b=9.2 \AA, c=10.5 \AA$ and $\alpha=$ 100 (Figure 10). The density of the monoclinic structure calculated on the basis of the lattice constants is about $1.37 \mathrm{~g} / \mathrm{cm}^{3}$

The X-ray diffraction data shows that the normal of the monoclinic (001) plane is parallel to the draw axis and that the monoclinic (010) plane is not perpendicular to the axis. That is, the monoclinic $a$ - and $b$-axes are perpendicular to the draw axis and the $c$-axis is tilted in the monoclinic $b_{\mathrm{m}} c_{\mathrm{m}}$ plane. The tilt angle of the $c$-axis is estimated at about $10^{\circ}$ from the distance between the two maxima of the split equatorial reflection of monoclinic 010 . Using the meridio- nal spacing of $10.3 \AA$, the monomer length is calculated to be $10.5 \AA$. Therefore, the monomer length is considered to be reduced by shrinkage of the monomer conformation.

The monoclinic structure is naturally disordered in the paracrystalline state. The paracrystalline 001 reflection is very sharp, while the equatorial reflections are broad. Accordingly, the disorder is mainly induced in the $a_{\mathrm{m}} b_{\mathrm{m}}$ plane. As the paracrystalline structure is formed intermediately in the growth of triclinic crystals from the amorphous state, it may be regarded as an aggregation of microscopic triclinic crystals in which the inclination direction of the plane of the binzene ring is different. In this case, the monoclinic state corresponds to the average of these triclinic crystals.

\section{Tilt of the Crystalline c-Axis in the Slowly}

\section{Drawn Sample}

The X-ray diffraction studies of slowly drawn samples showed that the triclinic crystallites are tilted in a manner known as the tilted orientation, and that the azimuth of the tilt varies with annealing temperature. The variation of the azimuth is shown in Figure 8, where the slope of the $\theta-T$ curve changes at about $240^{\circ} \mathrm{C}$. The tilt angle was about $8^{\circ}$ in the samples annealed at low temperatures and about $5^{\circ}$ in the samples annealed at high temperatures. The angle between the normal of the triclinic (001) plane and the draw axis was always decreased by the tilt.

In the case of annealing at low temperatures, the crystallites became triclinic after passing through the monoclinic paracrystalline state, and the triclinic crystallites were anisotropic in external form. In the case of annealing at high temperatures, a well-developed layer structure was observed by small angle X-ray diffraction, whereas the evidences of both the paracrystalline state and the anisotropy in external form of the triclinic crystallites disappeared in the wide angle patterns.

These results suggest that the causes of the tilting differ at low and high temperatures. At low annealing temperatures, the crystalline tilt is considered to arise in the course of the transition from the paracrystalline to the triclinic state. In the case of high temperatures, the tilt 
seems to be related to the formation of the layer shown in the previous section, the monoclinic structure. $c$-axis is tilted in the $b_{\mathrm{m}} c_{\mathrm{m}}$ plane $(\alpha=100)$. The Tilting at Low Annealing Temperatures. As transformation from the monoclinic to the tri-

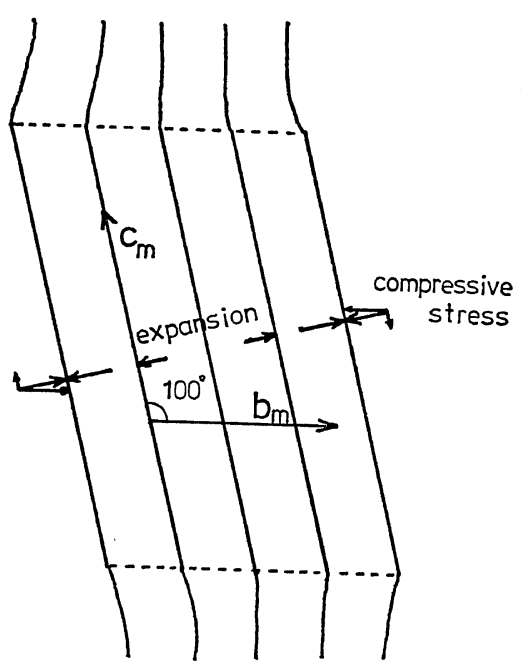

a

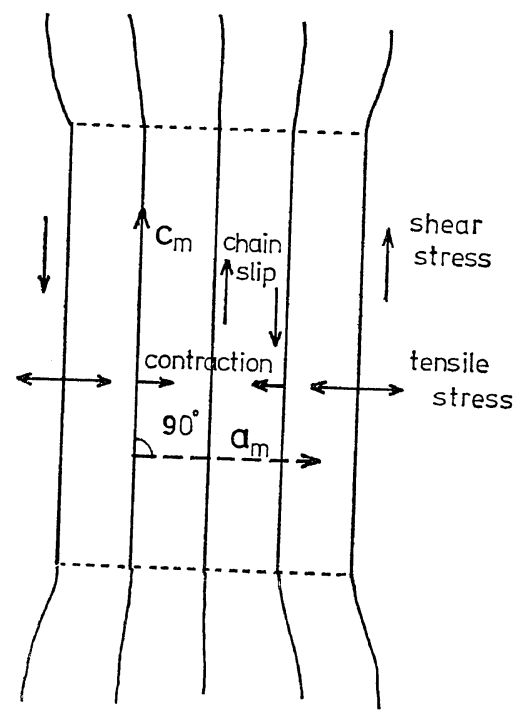

C

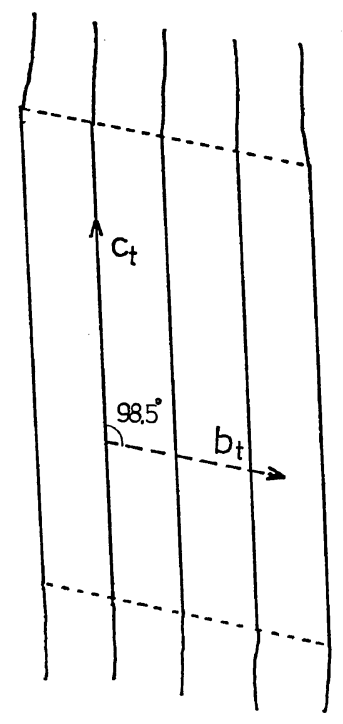

b

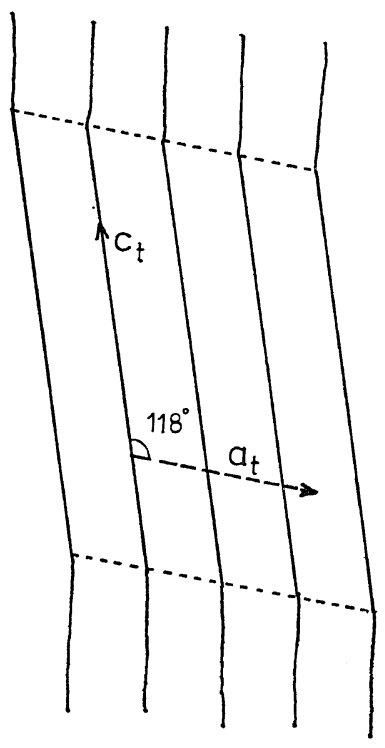

d

Figure 11. Internal stresses and crystalline tilt. (a) and (b) show the conditions in the monoclinic $b_{\mathrm{m}} c_{\mathrm{m}}$ plane (which corresponds to the triclinic $(\overline{2} 10)$ plane), (c) and (d) show the conditions in the plane perpendicular to the monoclinic (110) plane or to the triclinic (100) plane. 
clinic form requires expansion in the direction normal to the monoclinic (010) plane (see Figure $3 b)$. X-ray diffraction data of the sample annealed at $60^{\circ} \mathrm{C}$ actually indicate that the crystals do expand in this direction at the early stage of the transformation.

The transformation of the paracrystalline state into the triclinic one requires volume contraction of the crystallites, since the densities of the triclinic and the paracrystalline crystals are 1.455 $\mathrm{g} / \mathrm{cm}^{3}$ and $1.37 \mathrm{~g} / \mathrm{cm}^{3}$ respectively. As shown in Figure $3 b$, the contraction mainly arises in the spacing of the triclinic (100) plane.

At temperatures near or below the glass transition temperature, the molecular motion is strongly restricted and only local movement of molecules is allowed. Under these conditions, both expansion and contraction of a crystallite give rise to stresses between the crystallite and the amorphous part around it.

The expansion of the spacing of the monoclinic (010) plane in the course of the transition from the monoclinic to the triclinic state, which is considered to be caused by an ordering of the positions of the benzene rings, yields compressive stress parallel to the monoclinic $b_{\mathrm{m}} c_{\mathrm{m}}$ plane around the crystallits (Figure 11a). This stress can be relaxed by rotation of the crystallite in the following way. The stress is divided into two components in the directions shown in Figure 11a. One is shear stress parallel to the $c$-axis and the other is compressive stress normal to the $c$-axis. As the compressive components are equal on both sides of the crystallite, they balance each other and only the shear component effectively works on the crystallite. When the crystallits is rotated by the shear component, the chain tilt and the width of the crystallits in the direction normal to the $c$-axis decreases (Figure 11b), so that the compressive stress is relaxed. The decrease in the chain tilt in the $b_{\mathrm{m}} c_{\mathrm{m}}$ plane agrees with the result of X-ray observation. As the values of $\alpha$ in the monoclinic and the triclinic structures are $100^{\circ}$ and $98.5^{\circ}$ respectively, the transformation of the monoclinic into the triclinic structure requires little chain sliding in the $b_{\mathrm{m}} c_{\mathrm{m}}$ plane.

During the development of the triclinic crystallite, the spacing of the triclinic (100) plane is contracted. As the triclinic (100) plane transforms from the monoclinic (110) plane, the monoclinic crystal contracts in the direction normal to the (110) plane, as shown in Figure 11c. The contraction of the paracrystalline crystallite yields tensile stress perpendicular to the monoclinic (110) plane. On account of poor molecular mobility, the balance of forces seems to be unstable, hence the crystallite is easily

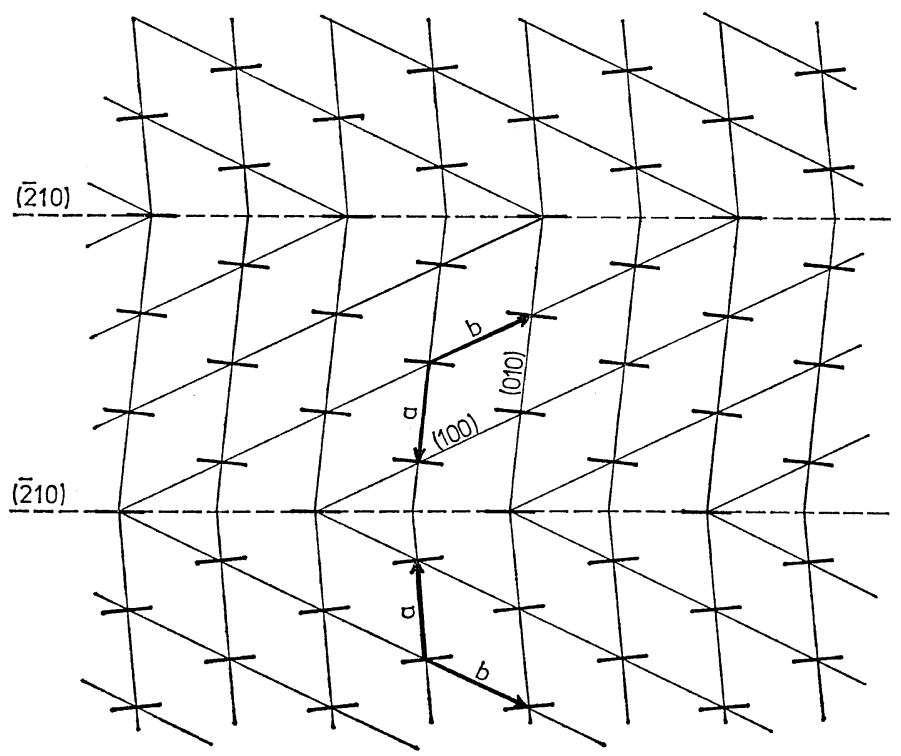

Figure 12. The $(\overline{2} 10)$ twinning of the triclinic crystallites. 
rotated in the plane of the paper in Figure 11c. On the other hand, the transition from the monoclinic to the triclinic state requires chain sliding in the $a_{\mathrm{m}} c_{\mathrm{m}}$ plane, because the triclinic $a$-axis is very much inclined $\left(\beta=118^{\circ}\right)$. The chain sliding causes shear stress on both sides of the crystallite, as shown in Figure 11c. Therefore, the crystallite will rotate so that the shear stress is relaxed, and consequently the chains and the triclinic (001) plane are tilted in opposite directions, in agreement with the result of X-ray observation (Figure 11d). The tilting of the $c$ - axis also reduces the tensile stress, since the lateral dimension of the crystallite is increased by the tilt.

From these considerations it is concluded that, in the course of the transition from the monoclinic to the triclinic state, the tilt of the $c$-axis decreases in the $b_{\mathrm{m}} c_{\mathrm{m}}$ plane and increases in the plane normal to the triclinic (100) plane and parallel to the $c$-axis. Accordingly, the azimuth of the tilt varies from the direction parallel to the monoclinic (100) plane $\left(\theta=100^{\circ}\right)$ towards that perpendicular to the triclinic (100)

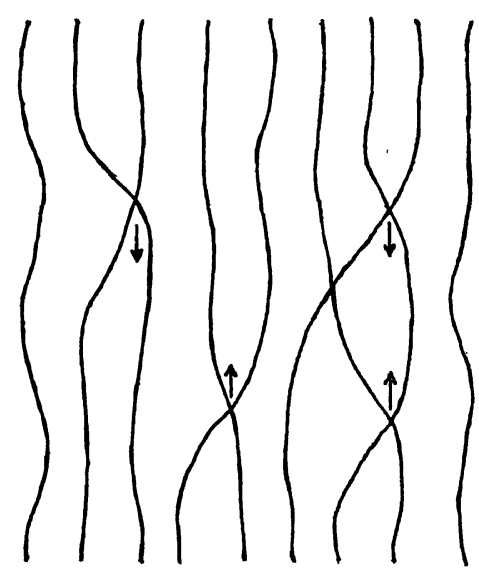

a

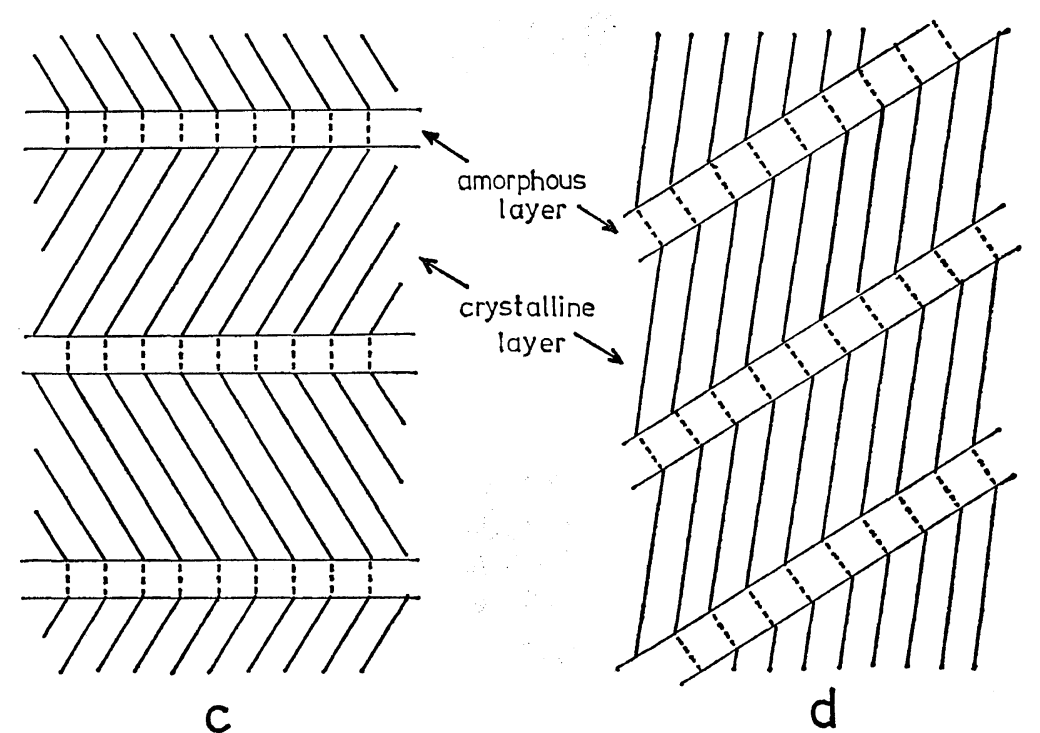

Figure 13. Layer structures in highly oriented material. (a) and (b) show the formation of layer structure, (c) and (d) show the possible layer structures. 
plane $\left(\theta=30^{\circ}\right)$, which shows close agreement with the variation observed in this experiment at low temperatures.

The triclinic crystallite produced by annealing at low temperatures is anisotropic in its external shape: that is, the crystallite develops in the direction nearly perpendicular to the $(0 \overline{1} 1)$ plane. In order to explain the anisotropy of the triclinic crystallite, we consider the possibility of twinning in the early stage of the transformation.

The monoclinic $a$-axis is the unique axis, so that the monoclinic $b_{\mathrm{m}} c_{\mathrm{m}}$ plane contains the molecular chains. The plane transforms to the triclinic (210) plane, remaining almost parallel to the draw axis, hence it is possible to form a twin whose twin plane is $(\overline{2} 10)$. The triclinic planes other than the $(\overline{2} 10)$ plane are not parallel to their corresponding planes in the monoclinic crystals. Therefore, it is difficult to form a coherent twin plane which coincides with any triclinic plane other than $(\overline{2} 10)$. In the case of the $(\overline{2} 10)$ twinning, crystallites easily grow along the $(\overline{2} 10)$ plane; the triclinic $0 \overline{1} 1$ reflection is sharpest, since the $(0 \overline{1} 1)$ plane is nearly perpendicular to the $(\overline{2} 10)$ plane (Figure 12).

Tilting at High Annealing Temperatures. At high temperatures, molecular mobility is high and wider crystallites may develop than those grown at low temperatures. When the crystallites have sufficiently developed to form a layer structure, the surface of the crystalline layer will coincide with a crystallographic plane so as to reduce the surface free energy.

In highly oriented polymers, crystallization will proceed by excluding disorders from the crystallizing region along the molecular axis (Figure 13a), and the disorders gather together to form amorphous regions (Figure 13b). As a result, the region left uncrystallized will expand laterally, while the crystallized region will contract. This discrepancy will give rise to stress between the crystalline and amorphous regions. As discussed in a previous paper, ${ }^{9}$ the stress is diminished by widening the chain interval on the surface of the crystalline layer. The condition is satisfied by tilt of the crystalline $c$-axis (Figure 13c) or by inclination of the layer surface (Figure 13d). The stress is also reduced by sharp folding of molecules at the layer surface, but the effect of folding seems to be ignored in cold drawn PET.

In the sample drawn at $0.1 \mathrm{~mm} / \mathrm{sec}$ and annealed at $250^{\circ} \mathrm{C}$ for $10^{3} \mathrm{sec}$, the inclination of the layer was about $25^{\circ}$, in agreement with that of the (001) plane of the triclinic crystal. If the layer surface is parallel to the (001) plane, the tilting of the crystalline $c$-axis is most effective in reducing the stress when the $c$-axis is tilted in the direction of the maximum slope of the (001) plane. The tilt line is then inclined to the $b^{*}$-axis at $40^{\circ}$. This value agrees with that obtained in this experiment.

In the case of high annealing temperatures, we do not know whether or not the triclinic state forms through the paracrystalline one. However, the continuous change of the tilt line seems to support the assertion that the paracrystalline order also appears, though for a very short time, at high annealing temperatures.

\section{Tilt of the Crystalline c-Axis in the Rapidly Drawn Sample}

The X-ray pattern of the rapidly drawn sample showed that strain-induced crystallization occurred in the course of the cold drawing, but paracrystalline reflections did not appear. Since the 010 reflection concentrated on the equator, the chains are not tilted. In annealing at low temperatures, the crystallization advanced very little and the $c$-axis did not deviate from the draw axis. The X-ray data of the samples annealed at high temperatures showed that the tilt of the crystalline $c$-axis is smaller, while the layer inclination is larger, than those of the slowly drawn samples.

In the case of rapid drawing, chain segments are strongly oriented, since the tensile stress acts on them without relaxation. Therefore, crystallization will advance considerably during the drawing. In this case, it is difficult for chains to tilt even when thermal motions are somewhat allowed by the annealing treatment. The fact that crystallization is advanced very little at low temperatures suggests that the tilt of the crystalline $c$-axis plays a significant role during crystallization.

At high temperatures, tilting of crystallites is considered to occur in nearly the same way as in the slowly drawn sample, since the value of 
$\theta$ varies almost in the same manner as for the slowly drawn samples. In rapidly drawn samples, the stress which works between the crystalline and amorphous layers seems to be diminished mainly by inclination of the layer surface.

\section{CONCLUSION}

In the case of slowly drawn PET, paracrystalline structure appears in the early stage of crystallization from the oriented amorphous state. The paracrystalline structure is formed on the basis of a monoclinic structure. It is thought that the crystallites become triclinic after passing through an intermediate monoclinic structure. This transformation is clearly observed at temperatures between $40^{\circ} \mathrm{C}$ and $100^{\circ} \mathrm{C}$.

The triclinic crystalline $c$-axis is always tilted by several degrees from the draw axis. The azimuth of the tilt, determined crystallographically in the unit cell, varies with annealing temperature. The azimuthal variation is explained by two possible mechanisms for the crystalline tilt. At low annealing temperatures, the tilt is related to the transition from the monoclinic structure. At high temperatures, the tilt is caused by the formation of the layer structure.
In the case of rapidly drawn PET, the triclinic crystal is formed during cold drawing, and the intermediate paracrystalline structure is not observed. The tilt of the triclinic $c$-axis is smaller and the layer inclination is larger than those measured in the slowly drawn samples.

Acknowledgement. The authors wish to thank Professor Y. Tajima of Tokyo Metropolitan University for many helpful discussions.

\section{REFERENCES}

1. R. Bonart, Kolloid-Z., 213, 1 (1966).

2. G. S. Y. Yeh and P. H. Geil, J. Macromol. Sci., B1, 235 (1967).

3. G. S. Y. Yen and P. H. Geil, ibid., B1 251 (1967).

4. R. de P. Daubeny, C. W. Bunn, and C. J. Brown, Proc. Roy. Soc. (London), Ser A, 226, 531 (1954).

5. R. Bonart, Kolloid-Z., 199, 136 (1964).

6. R. Bonart, ibid., 231, 438 (1968).

7. R. Bonart, ibid., 210, 16 (1966).

8. C. W. Bunn, H. S. Peiser, and A. Turner-Jones, J. Sci. Instrum., 21, 10 (1944).

9. T. Seto and T. Hara, Rep. Progr. Polym. Phys. Jap, 9, 207 (1966). 